\title{
Functional neuroanatomy of the insular lobe
}

\author{
C. Stephani - G. Fernandez-Baca Vaca • \\ R. Maciunas • M. Koubeissi · H. O. Lüders
}

Received: 8 September 2010/ Accepted: 25 November 2010/Published online: 14 December 2010

(C) The Author(s) 2010. This article is published with open access at Springerlink.com

\begin{abstract}
The insula is the fifth lobe of the brain and it is the least known. Hidden under the temporal, frontal and parietal opercula, as well as under dense arterial and venous vessels, its accessibility is particularly restricted. Functional data on this region in humans, therefore, are scarce and the existing evidence makes conclusions on its functional and somatotopic organization difficult. 5 patients with intractable epilepsy underwent an invasive presurgical evaluation with implantation of diagnostic invasive-depth electrodes, including insular electrodes that were inserted using a mesiocaudodorsal to laterorostroventral approach. Altogether 113 contacts were found to be in the insula and were stimulated with alternating currents during preoperative monitoring. Different viscerosensitive and somatosensory phenomena were elicited by stimulation of these electrodes. A relatively high density of electrode contacts enabled us to delineate several functionally
\end{abstract}

Electronic supplementary material The online version of this article (doi:10.1007/s00429-010-0296-3) contains supplementary material, which is available to authorized users.

C. Stephani - G. Fernandez-Baca Vaca - M. Koubeissi · H. O. Lüders

The Neurological Institute, Department of Neurology, University Hospitals Case Medical Center,

11100 Euclid Avenue, Cleveland, OH 44106, USA

\section{R. Maciunas}

The Neurological Institute, Department of Neurosurgery, University Hospitals Case Medical Center, 11100 Euclid Avenue, Cleveland, OH 44106, USA

\section{Stephani $(\bowtie)$}

Department of Clinical Neurophysiology, University Medical

Center Goettingen, University Hospital Goettingen,

Robert-Koch-Strasse 40, 37075 Goettingen, Germany

e-mail: CStephani@med.uni-goettingen.de distinct areas within the insula. We found somatosensory symptoms to be restricted to the posterior insula and a subgroup of warmth or painful sensations in the dorsal posterior insula. Viscerosensory symptoms were elicited by more anterior electrode contacts with a subgroup of gustatory symptoms occurring after stimulation of electrode contacts in the central part of the insula. The anterior insula did not show reproducible responses to stimulation. In line with previous studies, we found evidence for somato- and viscerosensory cortex in the insula. In addition, our results suggest that there is a predominantly posterior and central distribution of these functions in the insular lobe.

Keywords Insula Cortical maps · Somatosensory areas · Viscerosensation $\cdot$ Intracranial recording

\section{Introduction}

First described by Johann Christian Reil in the eighteenth century (1809), the insula has "long been a terra incognita for anatomists" (Penfield and Rasmussen 1950). It is completely covered by its neighboring cortical structuresthe frontal, the parietal and the temporal operculum. Macroscopically, the central sulcus of the insula divides it into an anterior and a posterior part (Fig. 4). The anterior part includes three short gyri-the anterior, middle and posterior short gyrus-as well as an additional accessory gyrus on the ventral margin of the anterior part of the insula. The posterior part has two long gyri-an anterior and a posterior long gyrus (Türe et al. 1999). Two (Brodmann 1909), three (von Economo and Koskinas 1925; Bailey and von Bonin 1951; Mesulam and Mufson 1985) or more (Vogt and Vogt 1919; Rose 1928; Kurth et al. 2010a) cytoarchitectonically distinguishable cortical 
areas have been described in the insula, depending on the pattern of lamination or myelination. Based on the degree of granularity, a tripartition into an anterior agranular cortex, an intermediate dysgranular and a posterior granular cortex is commonly referred to in modern descriptions of the insula (Mesulam and Mufson 1982a). According to studies in old world monkeys, the insula receives main afferents from the amygdala, the dorsal thalamus and different cortical regions, particularly the sensory cortices and the auditory cortex. Most of these afferents terminate in the posterior granular part of the insula whereas the ventral anterior agranular insula receives predominantly afferents from limbic cortex, e.g. the entorhinal, perirhinal, posterior orbitofrontal cortex and the cingulate gyrus. The efferents of the ventral anterior insula reciprocate the afferents of the anterior insula, which is not the case in the posterior insula (Mesulam and Mufson 1982b; Mufson and Mesulam 1982).

Information concerning the function of the insular lobe in humans is based-among other sources-on semiological data of patients with insular epilepsy, results of stimulation of intracerebral electrodes and neuroimaging studies (Isnard et al. 2004; Penfield and Rasmussen 1950; Kurth et al. 2010b).

A sequence of symptoms as characteristic of insular epilepsy has been derived from studies in several patients with drug-resistant epilepsy whose invasive electroencephalography (EEG) revealed a seizure onset within the insula (Isnard et al. 2004). Paresthesias of electricity or warmth, feelings of pharyngo-laryngeal constriction and dysphonic or dysarthric speech were described as typical of seizures that started from the insular cortex. But von Lehe et al. (2009) did not find such a typical semiological pattern in the Video-EEG of 24 patients with epilepsy and insular lesions on the magnetic resonance imaging (MRI).

Intraoperative electrocortical stimulation in a group of 36 patients with positive results at 82 separate stimulated points on the insula induced viscerosensitive or -motor and somatosensory symptoms that occurred throughout most parts of the insula, particularly in the more inferior anterior parts underlying the temporal operculum. No clear somatotopic distribution was found (Penfield and Faulk 1955). This study was complemented recently when predominantly somatosensory, viscerosensory or -motor and gustatory responses were reported after extraoperative stimulation from depth electrodes implanted radially in the insulae of 14 patients with epilepsy (Ostrowsky et al. 2000). Generally, somatosensory responses were more often induced with stimulation of the posterior insula and viscerosensory responses more often in the anterior insula. Then, in an expanded group of 50 patients, diverse somatosensory (warmth sensation, electric current sensation, painful paresthesias), viscerosensitive (pharyngolaryngeal constriction, abdominal heaviness, thoracic constriction, nausea), auditory, dysarthric, olfactogustatory symptoms or whole body sensations or sensations of unreality were described but were widely distributed within the insula (Isnard et al. 2004). In a similar recent series of ten patients with intractable epilepsy, somatosensory responses prevailed again, including sensations of numbness, tingling, warmth, pain or electric current. In addition, viscerosensation like nausea and "buzzing in the abdomen" was recorded while motor association responses, auditory responses, vestibular responses and language or speech responses occurred less frequently (Nguyen et al. 2009).

Numerous studies performing neuroimaging of the insula do confirm and expand the previously introduced evidence (Kurth et al. 2010b). Predominant activation of insular cortex after tasks including somatosensory stimulation (Ruben et al. 2001), thermosensory stimulation (Brooks et al. 2005; Craig et al. 2000), viscerosensory stimulation (Wang et al. 2008), autonomic stimulation (Pollatos et al. 2007) and gustatory stimuli (Small et al. 2003) has been observed using functional magnetic resonance imaging (fMRI) and positron emission tomography (PET). Results of these and other studies and considerations concerning the neuroanatomy of the insula have led to a hypothesis regarding a central role of the insula for higher cortical functions and processing of homeostatic information (Singer et al. 2004; Craig 2009).

Using invasive electrocortical stimulation of the insula in patients with refractory epilepsy, our study presents further evidence for a distinct functional organization of the human insula.

\section{Methods}

\section{Subjects}

Five patients (all female, median age 40.2 years) with intractable epilepsy underwent invasive monitoring in the presurgical evaluation for intractable epilepsy in our Video-EEG monitoring unit between March and May 2009. These patients received implantations of hippocampal, parahippocampal, orbitofrontal and insular depth electrodes. The right insula was covered in two patients, the left insula in two patients and bilateral insular electrodes were placed in one patient. The decision for the number and location of invasive electrodes was taken in an interdisciplinary presurgical conference based on anamnestic, semiological, electroencephalographic and imaging data.

During the course of the invasive recording, stimulation of selected electrode contacts, including all contacts within 
the insula, was performed to delineate cortical function and to determine whether seizure-like symptoms could be induced by stimulation. At the time of stimulation, all patients had been tapered off medication (Table 1).

\section{Procedure}

Electrodes were implanted stereotactically under general anesthesia. Planning of the procedure included a simulation of implantation trajectories with iplan-stereotaxy $2.6^{\circledR}$ software (Brainlab, Munich, Germany) based on recent 3-T MRI images of the brain. Insular electrodes were implanted using a mediodorsal to lateroventral technique with insertion at the superior lateral surfaces in the rolandic area. Electrodes had 10-12 contacts each with a diameter of $1 \mathrm{~mm}$ and an interelectrode distance of $2.5 \mathrm{~mm}$. The surface of each electrode contact was $1.96 \mathrm{~mm}^{2}$. Three depth electrodes were implanted in each insula to cover the anterior, middle and posterior portion of the insula. X-Ray and cranial computer tomography (CT) were performed within $24 \mathrm{~h}$ postsurgically. Using iplan-stereotaxy $2.6^{\circledR}$ software, postsurgical cranial CT and presurgical brain MRI were superimposed for relatively precise localization of single electrode contacts within the patient's presurgical MRI.

\section{Stimulation}

Electrical stimulation was applied with an Ojemann ${ }^{\circledR}$ current stimulator. Stimulation paradigms included a stimulation frequency of $50 \mathrm{~Hz}$, pulse width of $0.5 \mathrm{~ms}$, duration of stimulation train between 3 and $5 \mathrm{~s}$ and stimulation intensities of 1.5-14 mA. Stimulation was biphasic. Therefore, we multiplied the stimulation intensity as shown on the display of the stimulator by two, taking into account the positive and the negative phase of the stimulus. Hence, all stimulation intensities reported here are twice those of the stimulation intensities displayed on the stimulator.
Starting with a stimulation intensity of $1.5 \mathrm{~mA}$, we increased the intensity in steps of 1-2 mA until $14 \mathrm{~mA}$ or induction of a symptom. Stimulation was repeated several times if the initial stimulation produced any symptoms. Stimulation was not repeated when the first stimulation produced no symptoms or signs. We did not screen for negative motor symptoms or speech disturbance during stimulation in this study. All contacts that elicited the same or similar responses after stimulation at least twice were considered as "positive" electrodes. Electrodes that showed reproducible responses at least twice but not in $100 \%$ of all stimulations were also considered "positive" electrodes (Table 2).

In order to establish maps of insular function from single patients, we produced a simple scheme of the insula including main insular landmarks. Main landmarks like the two posterior long gyri of the insula were clearly recognizable in every patient as revealed by visual inspection of the superimposition of the postsurgical CCT on the presurgical cranial MRI. Based on the location of the "positive" electrode contacts relative to these landmarks, we combined responses of the different patients in a single scheme.

\section{Statistics}

To test for differences in stimulation intensities between patients as well as between groups of responses, we applied a univariate analysis of variance $(\alpha=0.05)$ with stimulation intensity being the dependent variable.

\section{Approval}

The study protocol was designed according to the declaration of Helsinki and has been approved by the local Institutional Review Board (IRB) committee of the Case Western Reserve University. Informed consent was obtained from all patients included in this report.

Table 1 Patient characteristics and epilepsy classification

\begin{tabular}{llllll}
\hline Patient & Hdn & Age & Age of onset & Epileptogenic zone & Semiology \\
\hline 1 & $\mathrm{R}$ & 32 & 1 & Multiregional & (1) Gustatory Aura $\rightarrow$ Hypermotor seizure (LOC) \\
& & & & (2) Abdominal Aura $\rightarrow$ Automotor seizure \\
2 & $\mathrm{R}$ & 52 & 44 & (3) Abdominal Aura \\
3 & $\mathrm{R}$ & 58 & 32 & Right orbitofrontal lobe & Automotor seizure (LOC) $\rightarrow$ GTCS \\
4 & $\mathrm{R}$ & 38 & 36 & Right temporal lobe & Dialeptic seizure $\rightarrow$ GTCS \\
5 & $\mathrm{R}$ & 21 & 16 & Left posterior temporal lobe & Abdominal Aura $\rightarrow$ Dialeptic seizure $\rightarrow$ GTCS \\
\hline
\end{tabular}

Hdn handedness, $R$ right, LOC loss of consciousness, GTCS generalized tonic-clonic seizure 
Table 2 Statistics of responses to electrocortical stimulation

\begin{tabular}{llllll}
\hline Electrode contacts & AI & MI & PI & All & $\%$ \\
\hline Electrode contacts within the insula & 10 & 52 & 51 & 113 & 100 \\
Responses within the insula & 0 & 26 & 36 & 62 & 55 \\
Responses confirmed at least twice in one & - & 22 & 32 & 54 & 48 \\
$\quad$ session & - & & & & \\
Responses confirmed in a second session & - & 16 & 13 & 29 & 26 \\
\hline
\end{tabular}

$A I$ anterior insula, $M I$ middle insula, $P I$ posterior insula

\section{Results}

Stimulation of 62 out of 113 contacts $(55 \%)$ located within the insula produced responses. However, only 54 (48\%) of the 62 responses were reproducible at least once and only $29(26 \%)$ were found to be convincingly reproducible during a separate second stimulation. The mean stimulation intensity necessary to induce a clinical response in these 53 electrode contacts was $9.15( \pm 2.2) \mathrm{mA}$ and differed significantly neither between the different categories of responses $(p=0.072)$ nor the different patients $(p=$ $0.797) .37$ out of 54 contacts were clearly allocated in the grey matter (Fig. 1), 9/54 contacts were located in the grey-white matter transition (Fig. 2) whereas 8 out of 54 contacts were more likely to be located in the intragyral or subinsular white matter (Fig. 3). These eight contacts were included in our assessment since they were in functional continuity with their surrounding electrode contacts in the grey matter.

Even though the number of contacts within the right and the left insula was similar (right insula $=61$, left insula $=52$ ) reproducible responses were more frequently elicited within the left insula (left insula $=39$, right insula $=15$ ). There were also differences concerning the responsiveness of different insular regions. We divided the insula into three regions. A posterior insula caudal to the postcentral sulcus of the insula, a middle insula between the postcentral sulcus of the insula and the precentral sulcus of the insula and an anterior insula cranial from the precentral sulcus of the insula (Fig. 4). The likelihood of producing reproducible responses was highest within the posterior insula $(32 / 51=63 \%)$, decreased in the middle part of the insula $(22 / 52=42 \%)$ and was lowest with the anterior insula electrodes $(0 / 10=0 \%)$. Supplement 1 and 2 (Online Resource) provide a graph and a list of each patient's responses to electrocortical stimulation.

Symptoms elicited by stimulation of the insula can be divided into two main categories: visceral or internal sensation on the one hand $(n=24)$ and somatosensation on the other $(n=30)$. Almost without exception, electrode contacts that produced visceral responses were located
Fig. 1 Presurgical MRI and postsurgical cranial CT were superimposed using the software Brainlab ${ }^{\circledR}$. Then each single electrode contact was located and displayed on three planes. One electrode contact is indicated by an arrow
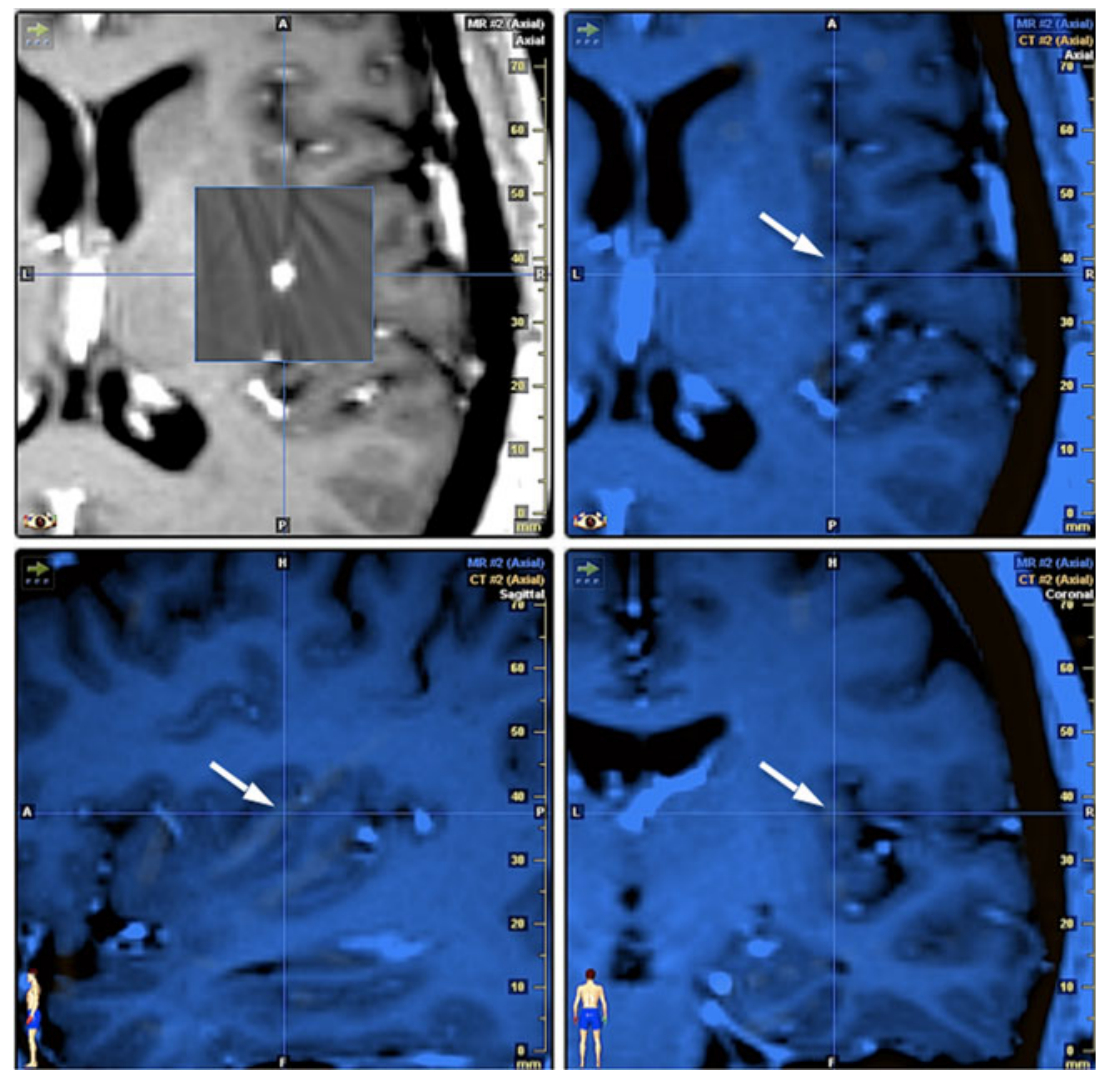
Fig. 2 Superimposition of a postsurgical cranial CT on a presurgical MRI using the software Brainlab ${ }^{\circledR}$. Example of an electrode contact in the greywhite matter transition (indicated by an arrow)
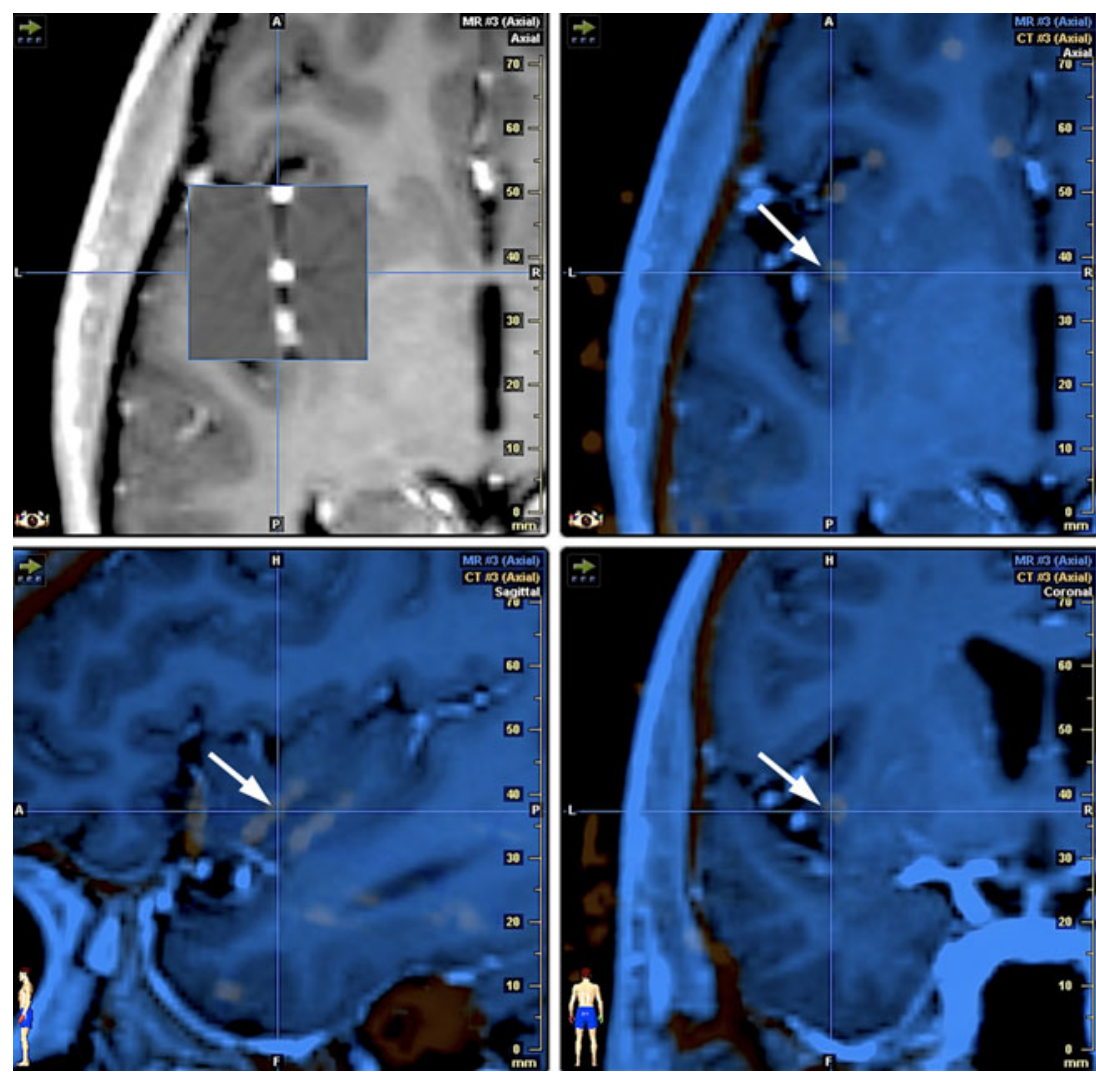

anterior to somatosensory responses, which were found in the most posterior part of the insula.

Within the group of visceral sensations, taste phenomena represented a separable group of special viscerosensation. Nine electrodes, exclusively located in the central part of the insula, produced taste phenomena. Often these were unpleasant phenomena described as "bad", "nasty", "nautious" taste $(n=7)$. In $n=3$ electrodes, taste sensation was qualified as "metallic" or "like aluminum". Localization of taste sensation included the whole oral cavity in the back of the mouth, the back of the tongue or even in the back part of the nose. The taste sensation was lateralized at seven electrodes and was always ipsilateral to the side of stimulation [Fig. 5; supplement 1 and 2 (Online Resource)].

Responses elicited at eight electrodes were classified as general viscerosensation and were described as a feeling of "throwing up", having "something in the throat", "vibration in the stomach" or simply "abdominal sensation" [Fig 6; supplement 1 and 2 (Online Resource)]. In six of these contacts, the patients made some comment suggesting a visceral movement ("feeling of throwing up", "throat feels like shaking", "vibration in the stomach") but we were unable to observe any visceral movements.

Description of responses to stimulation from six electrode contacts could not be assigned to one distinct category of internal sensation. The feeling of a "dry sensation in the nose" was reported at two contacts and represents a more complex internal sensation that we could classify neither as being clearly gustatory nor as viscerosensory. In two other cases, the quality of the response changed even though the location of the response did not (stimulation of one electrode induced the feeling of "dropping of the mouth" the first time and elicited a "bad taste" when stimulated again; stimulation of another electrode produced a sensation of "something in the throat" the first time and of an unspecified "bad taste at the back of the throat" when repeating the stimulus). These electrode contacts are shown in a composite color code in Figs. 5 and 6.

Within the group of somatosensory responses, we distinguished sensations of warmth or pain from general somatosensations. Ten electrode contacts exclusively located in the posterior insular cortex elicited symptoms of warmth or pain in different parts of the body. Responses of warmth ( $n=6$ contacts in 2 patients) were located more ventrally and painful phenomena more dorsally ( $n=4$ in 2 patients) within the posterior insula after stimulation. The sensation of warmth was described as a "warm" or "hot" feeling in different parts of the body. There was no description of a cold feeling induced by stimulation of the posterior insula. Painful sensations could be described as "burning", "stinglike" or simply "painful". Sensations of warmth or painful sensation were always on the contralateral side of the body [Fig. 7; supplement 1 and 2 (Online Resource)]. 
Fig. 3 Superimposition of a postsurgical cranial $\mathrm{CT}$ on a presurgical MRI using the software Brainlab ${ }^{\circledR}$. Example of an electrode in the subinsular white matter (indicated by an arrow)
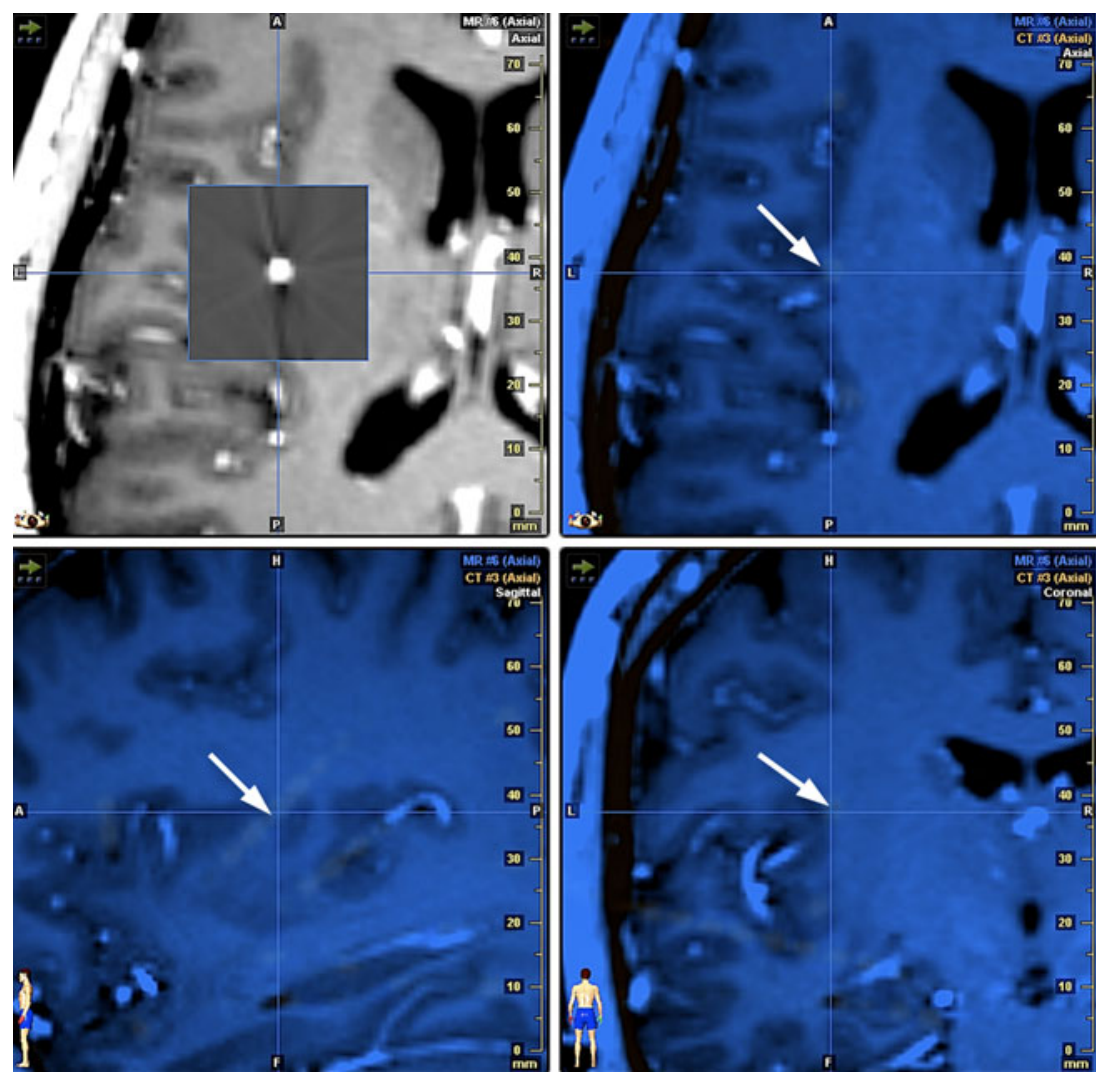

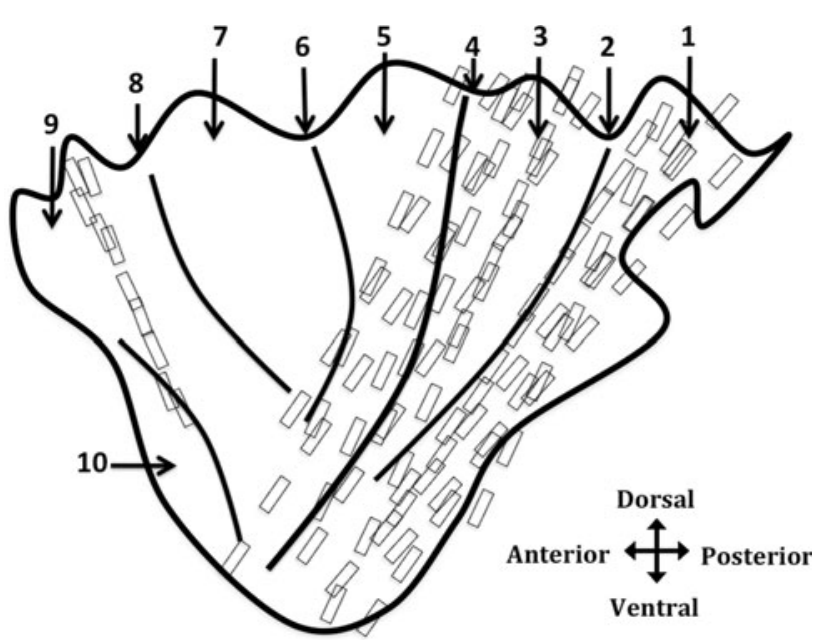

Fig. 4 Scheme of the insula. Included are the locations of all 113 contacts that were allocated in the insula after superimposing the postsurgical cranial CT on the presurgical MRI. Anatomical landmarks of the insula are indicated by numbers as follows: 1 posterior long gyrus of the insula, 2 postcentral insular sulcus, 3 anterior long gyrus of the insula, 4 central insular sulcus, 5 Posterior short gyrus of the insula, 6 precentral insular sulcus, 7 middle short gyrus of the insula, 8 short insular sulcus, 9 anterior short gyrus of the insula, 10 accessory gyrus of the insula

Four of these ten contacts elicited different responses when stimulated repeatedly. In these contacts, the qualitative dimension of the response elicited by stimulation changed, e.g. from "warm" to "numb", "painful" to "tingling" or "painful" to "pulling" sensation in a certain body part. In two of the electrodes, the location of the sensation also changed from leg to foot or arm to thigh. These electrode contacts are shown in a composite color code in Figs. 7 and 8.

General somatosensory responses $(n=20)$ were less well defined. They were described as "tingling", "feeling of pulsation", "feeling of vibration", or "feeling of numbness" in different body parts contralateral to the side of stimulation. Two electrode contacts in the most ventral part of the posterior insula elicited whole body sensations. All other somatosensory responses were lateralized to the side contralateral to the stimulated hemisphere.

To conclude, specific and non-specific somatosensations localized in the posterior-dorsal area of the insulaimmediately posterior to viscerosensory sensations and gustatory responses-were elicited [Fig. 8; supplement 1 and 2 (Online Resource)]. Stimulation of the anterior insula remained asymptomatic.

\section{Discussion}

This study of electrocortical stimulation shows four qualitatively and spatially distinct functional areas in the human 
Figs. 5-8 The color-coded pictograms of the insula include the localizations of those electrode contacts that evoked clinical responses with electrocortical stimulation. The responses were grouped into gustatory responses (Fig. 5), viscerosensory responses (Fig. 6), responses of warmth or pain (Fig. 7) and into general somatosensory responses (Fig. 8). The following color code is applied: blue gustation, yellow viscerosensation, red thermosensation, red with mark pain, green somatosensation. Composite color bars indicate qualitatively inconsistent or ambiguous symptoms after stimulation
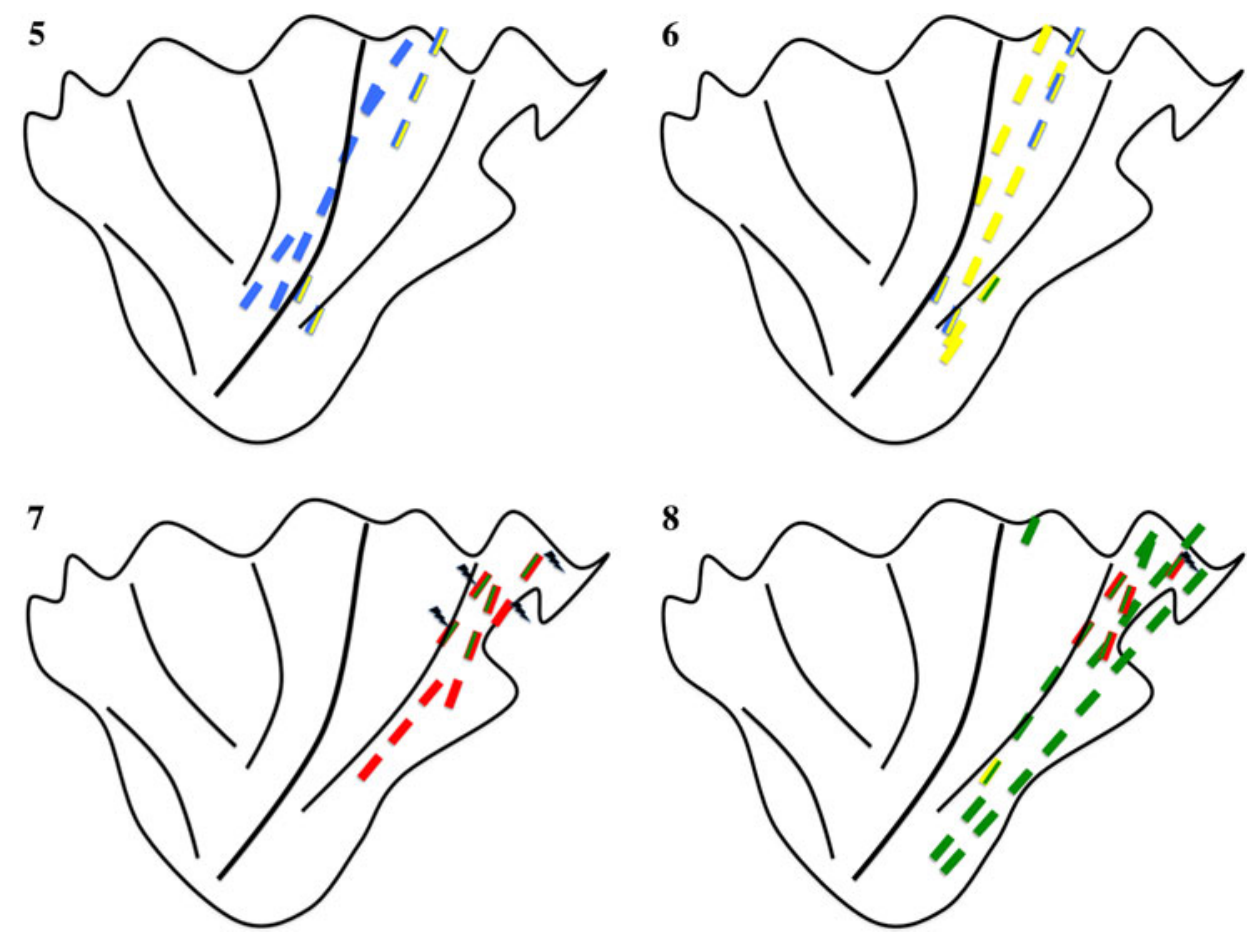

central and posterior insular lobe: phenomena of general somatosensation, thermal and pain perception, viscerosensation and gustation were elicited repeatedly when stimulating within the central and posterior insula. These results are consistent with neuroanatomical and neurofunctional data derived from animal as well as human studies and reveal distinct functions represented in distinct parts of the insular lobe. When Penfield and Faulk (1955) reported on their results of intraoperative surface stimulation of the insula, the most common responses were characterized as viscerosensory $(n=32)$ or somatosensory $(n=30)$ symptoms. This closely resembles the categorization of our results and their ratios (viscerosensory responses $n=24$, somatosensory responses $n=30$ ). Nevertheless, even if the stimulation paradigms were comparable to those used in our study, stimulation in Penfield and Faulk's study was confined to insular regions normally covered by the temporal operculum. Besides, Penfield and colleagues stimulated all electrodes at constant stimulus intensity and did not control for the occurrence of after-discharges. Still the degree of comparability between their results and more recent studies based on intracranial extraoperative electrodes is very high, suggesting that the possible bias due to spread of excitation or after-discharges was minimal. Nevertheless, the symptoms elicited in their study were more widely distributed and arose from stimulation of posterior, middle and anterior parts of the accessible insula. Despite the fact that Ostrowsky et al. (2000) show a tendency to discriminate between somatosensory symptoms and viscerosensory symptoms between more posterior and more anterior insular areas, respectively, more widespread patterns were also described in other recent studies (Ostrowsky et al. 2002; Isnard et al. 2004; Nguyen et al. 2009), suggesting that spread of excitation-a possible confounder in any study of electrocortical stimulation-may have contaminated the results. Therefore, agreement on functional representation in the insula does not translate to agreement on functional localization within the insula so far.

\section{Thermosensation and nociception}

Ostrowsky et al. (2002) found the representation of 15 painful insular responses to electrocortical stimulation in 14 patients to be located in the upper posterior insular cortex, predominantly in the right hemisphere. In addition, they found somatosensory responses and especially sensation of non-painful warmth to be more frequently located in the lower posterior insula. And a somatotopic distribution of painful responses to electrocortical stimulation of the posterior insula was demonstrated recently with responses in the upper limbs being more dorsal compared to those in the lower limbs and painful responses in the face being more rostral to those in the limbs (Mazzola et al. 2009). The distribution of our limited number of six sensations of warmth and four sensations of pain (each being exclusively located in the posterior insula) confirm these results. In our study, painful responses were elicited within the right as well as the left insula. However, concerning laterality and somatotopy, four responses is too small a number on which to base a definite conclusion. Moreover, our results are in good agreement with anatomical and 
histological studies in humans and animals. Fifty percent of those peripheral nerve fibers that transmit stimuli of warmth, pain and prick sensation have their first relay in lamina I within the dorsal horn of the spinal cord. This information, after crossing segmentally, is transmitted via the spinothalamic tract to its second relay at a specific subnucleus within the posterior ventromedian nucleus (Vmpo) of the thalamus (Craig et al. 1994). The cortical projection of this nucleus is thought to be within the posterior insula. This is an important deviation of the main somatosensory projections to the postcentral gyrus of the lateral hemisphere and maintains the common systematic neuroanatomic differentiation of an epicritic and a protopathic afferent somatosensory system as established on a spinal level. Several studies using fMRI found evidence for a somatotopic representation of pain in the dorsal posterior insula as well (Brooks et al. 2005; Henderson et al. 2007, 2010). Evoked responses after painful stimuli of the skin have been recorded with intracranial depth electrodes in the suprasylvian operculum after 140-170 ms and in a deeper insular area after 180-230 ms (Frot and Mauguière 2003). Confirming studies with scalp electrodes (Valeriani et al. 2000) or subdural electrode grids (Lenz et al. 1998) localized evoked responses after painful stimulation to the Sylvian region. Furthermore, Frot et al. (2007) demonstrated that peripheral thermal stimuli produced intensityrelated evoked potentials in the parietal operculum, whereas painful thermal stimuli predominantly produced evoked potentials in the posterior insula. Lesion studies also provide evidence for the importance of the posterior insula for pain processing (Biemond 1956; Birklein et al. 2005). In a study of six patients with heterogeneous parasylvian lesions on the MRI, elevated pain thresholds contralateral to the lesion measured by contact heat and pinprick pain were found only in patients whose lesions included the posterior insula and the parietal operculum. In the context of their results, the authors highlighted the significance of the parietal operculum and the insula for pain perception (Greenspan et al. 1999). In contrast, another study on patients with insular lesions found more heterogeneous effects of pain and temperature perception and argued for a modulatory role of the insula in connection with these qualities (Starr et al. 2009). However, temperature sensation was represented in the insula in a PET study, which showed activation of the middle/posterior insula that correlated with graded cooling. In addition, the authors proposed that the central pain syndrome after lesions including this anatomical region may be due to a "loss of the normal inhibition of pain by cold" (Craig et al. 2000). In our patients, the discrimination of painful responses was not very definite, and included a "stinglike" or a burning sensation. On the other hand, thermal responses were clearly identified by the patients in our study, providing convincing evidence for representation of thermosensation in the posterior insula. Given the gradual transition between non-painful and painful (thermo)sensation our results do not allow a more detailed delineation between these sensations.

\section{Somatosensation}

Penfield and Faulk (1955) reported on the high similarity between somatosensory responses to electrocortical stimulation of the insula and those that could be elicited by stimulation of the upper bank of the Sylvian fissure; this group first proposed "neighbourhood activation" as one potential explanation of these results (Penfield and Faulk 1955). However, subsequent studies repeatedly confirmed somatosensory responses after stimulation of the insula, supporting the hypothesis of an independent insular somatosensory area (Penfield and Faulk 1955; Ostrowsky et al. 2000; Isnard et al. 2004; Nguyen et al. 2009). Nevertheless, the functional significance of general somatosensory representation in the insula remains unclear and the concept of higher-order somatosensory areas may be relevant in connection with these results. Recent cytoarchitectonic studies argue against the somatosensory areas of the insula being simply an elongation of the secondary somatosensory cortex of the parietal lobe, especially, the parietal operculum. Advanced definitions of secondary somatosensory areas have been proposed by new cytoarchitectonic and neuroimaging studies in humans (Eickhoff et al. 2006, 2007) as well as evoked potential studies in non-human primates (Coq et al. 2004). Based on these studies, four different somatosensory maps have been delineated in the parietal operculum alone-each representing a complete body map. In this scheme, the second somatosensory area may be analogue to a dorsal posterior parietal opercular area (OP1), and a more anterior cytoarchitectonic area (OP4) may represent the somatosensory parietal-ventral area (PV). Two more ventral parietal opercular areas named OP2 (ventral posterior parietal operculum) and OP3 (ventral anterior parietal operculum) may be the neuroanatomical correlates of two ventral somatosensory areas of the parietal operculum as defined in the New World titi monkey that were termed rostral ventral somatosensory area and caudal ventral somatosensory area (Coq et al. 2004). Whereas previous cytoarchitectonic maps of the insula were mainly derived from non-human primates or based on single human individuals (Mesulam and Mufson 1982a; Brodmann 1909), in a recent study analyzing the cytoarchitecture of 10 post-mortem brains with an observer-independent method, three distinct cytoarchitectonic areas in the posterior insula were defined (Kurth et al. 2010a). Two granular cortical areas in the dorsal posterior insula named $\operatorname{Ig} 1$ and $\operatorname{Ig} 2$ and one 
dysgranular cortical area in the ventral posterior insula named Idg1 were delineated and may correlate to a variety of somatosensory responses to electrocortical stimulation of the insula in our as well as previous studies. Granularity in cytoarchitectonic classifications is typical of cortex with predominant afferents like the primary somatosensory cortex of the lateral surface of the brain. Indeed, the locations of Ig1 and Ig2 correspond clearly to those areas where general somatosensory and thermosensory/nociceptive responses were found in our study. Whereas an exact correlation cannot be derived from our study, Ig1 most closely corresponds to the responses of warmth or painful responses. General somatosensory responses were found within areas corresponding to $\operatorname{Ig} 1$ and $\operatorname{Ig} 2$. Interestingly, it was demonstrated by use of retrograde and anterograde axonal transport methods in macaque monkeys that the secondary sensory area $(\mathrm{S} 2)$ in the lateral sulcus of the brain, which itself receives mechanoreceptive somatosensory input from the primary sensory areas, is reciprocally connected to granular and dysgranular insular areas (Friedman et al. 1986). Moreover, projections of the granular and dysgranular insula to limbic areas like the amygdaloid complex and the entorhinal cortex suggest that these insular areas may be an important corticolimbic relay within a hierarchical network subserving tactile learning and memory. At least the aforementioned projections may explain the mechanoreceptive responses after stimulation of the posterior insula. Therefore, it may well be that the granular insular cortices represent secondary or tertiary general somatosensory areas whose functions have yet to be named (Kurth et al. 2010a). However, concerning thermosensation and nociception, the posterior insula may be part of the primary cortical representation of these functions given current functional neuroanatomic findings (Craig et al. 2000).

\section{Viscerosensation}

Viscerosensory symptoms represent the second major group of symptoms that were produced by stimulation of the insular cortices, very like previous studies with electrocortical stimulation (Penfield and Faulk 1955; Ostrowsky et al. 2000; Isnard et al. 2004; Nguyen et al. 2009). In fact, in an early report on electrocortical stimulation of the insula, one-third of all responses were related to the "abdominal cavity" (Penfield and Faulk 1955). The same authors proposed that the insula may be part of an oroalimentary cortex extending from the ventral precentral gyrus to the ventral insular cortex. In addition to reporting viscerosensory responses, they also recorded changes of gastric motor activity after stimulation of electrode contacts in a subgroup of four patients who agreed to intraoperative gastrographic recordings. Recorded gastric motor activity upon stimulation then was or was not accompanied by a feeling of gastric movements. Consequently, some of the viscerosensory responses of our patients during insular stimulation suggesting gastric or visceral movements may in fact have represented visceromotor responses. Changes of gastric motility after electrocortical stimulation of the insula have also been recorded invasively in macaque monkeys (Hoffman and Rasmussen 1953). In this study, unspecific gastrointestinal responses to cortical stimulation could be excluded by stimulation of the lateral hemisphere, which did not produce changes in gastrointestinal motility. Importantly, these effects were abolished after sectioning both vagal nerves. Indeed, ascending projections of the visceral organs terminate in the granular and dysgranular parts of the insula via the parvocellular nuclei of the lateral and medial ventroposterior thalami as demonstrated by evoked potential and neuronal labeling studies in rats (Cechetto and Saper 1987; Allen et al. 1991). Functional neuroimaging studies, e.g. after gastric distension in healthy volunteers, repeatedly also revealed that the subsequent metabolic or vascular activation predominates within or at least includes insular cortex (Vandenbergh et al. 2005; Ladabaum et al. 2007). The role of the insula for visceral motility may be further substantiated by reports of circumscribed insular lesions that produced isolated dysphagia - a common symptom after ischemic strokes (Stickler et al. 2003; Riecker et al. 2009). This may be considered evidence for the hypothesis of the insula being the central cortical projection of the nucleus of the solitary tract (NST), one important relay of afferent vagal nerve fibers (Saper 1982).

\section{Gustation}

The neuroanatomical pathway of taste processing has been well studied in animals. From taste buds gustatory information is transferred to the nucleus of the solitary tract (NST) via the chorda tympani and the greater superior petrosal branches of the facial (VIIth), the lingual branch of the glossopharyngeal (IXth) and the superior laryngeal branch of the vagal (Xth) nerve. These nerve fibers are arranged topographically from rostral to caudal with the facial nerve endings being in the rostral parts of the NST and the vagal nerve fibers ending in its caudal part. After the first relay of the taste neurons, gustatory information travels to the hypothalamus and the parvocellular ventromedian nucleus (VPMpc) of the thalamus in primates (Van Buren and Borke 1972). In rodents, gustatory information is processed via an additional relay in the brain stem called the pontine parabrachial nucleus, which then has a bipartite projection to subcortical nuclei as well as cortical areas (Small 2010). Whereas these pathways of the taste system are well established, the location of the primary gustatory 
cortex is less precisely defined (Kaas 2005; Small 2010). It is generally accepted that gustatory representation is not part of the primary somatosensory representation of the tongue as confirmed by extensive records of intraoperative electrical stimulation (Penfield and Rasmussen 1950; van Buren 1983). Our results indicate representation of taste information in the middle or central parts of the insula. This is consistent with the evidence that primary afferents from the VPMpc proceed to insula as well as frontal and parietal operculum (Pritchard et al. 1986). And even though the primary gustatory cortex in non-human primates has been assigned to the anterior insula, recent evidence from neuroimaging studies suggests that gustatory information may be represented "further caudally in the human compared to the monkey insular cortex" (Small 2010). Usually, taste phenomena were described as unpleasant or very unpleasant sensations. This strong affective component is in line with data from stimulation as well as description of seizures with gustatory phenomena (Penfield and Faulk 1955; Hausser-Hauw and Bancaud 1987). In addition, the facial expression of disgust was shown to particularly activate insular cortex in a neuroimaging study (Phillips et al. 1997). In our study, in 7 out of 14 contacts taste phenomena were found to be ipsilateral to the side of stimulation. Benjamin and Burton (1968), who stimulated the chorda tympani and the lingual-tonsillar branch of the glossopharyngeal nerve of the squirrel monkey, produced ipsilaterally but not contralaterally evoked potentials in the anterior opercular-insular cortex. Deficits in the qualitative and quantitative discrimination of gustatory stimuli were found on the side of the tongue ipsilateral to insular lesions (Pritchard et al. 1999). In addition, a bilateral deficit in taste recognition was reported in patients with left insular lesions in this study. Our results strongly support the predominantly ipsilateral cortical representation of taste in humans. This supports the hypothesis that the central representation of the solitary tract nucleus lies in the insula as described above.

\section{Further considerations}

Some comment is required regarding the discrepancies between this study and previous reports on insula stimulation. In clear contradiction to previous stimulation studies, stimulation of the anterior insula did not lead to any responses in our study. Still, even though 10 electrode contacts in the rostral part of the anterior insula were stimulated, the central part of the anterior insula was not covered with depth electrodes in this study. It is not clear if the anterior insula should be defined based on cytoarchitectonic characteristics or macroscopic divisions. It is of note that the central sulcus of the insula as a macroscopic border may not coincide with any microscopic parceling of the insula and hence may not be sufficient for functional segregation (Zilles and Amunts 2010). The anterior agranular insula shares characteristics with and is highly connected to limbic and paralimbic cortex based on studies in Old World monkeys (Mesulam and Mufson 1982b). The proposed homologue cortical areas in humans are often less responsive to electrocortical stimulation which may explain the lack of symptoms after stimulation of the anterior insula in our study. On the other hand, a participation of the insula in the perception and processing of subjective feelings, emotion and self-awareness has been proposed (Craig 2002) and it may be speculated that, given the close representations of taste, visceral perception, thermosensation, nociception and somatosensation in our study, the anterior parts of the insula may well be involved in more integrative functions relevant for homeostasis or emotional processing (Dupont et al. 2003; Naqvi et al. 2007; Craig 2009). It is possible that these functions may not be readily evoked by electrocortical stimulation.

In addition, it was surprising that in this study stimulation of the insula elicited no representation of autonomic signs. Significant changes in heart rate were neither detected after stimulation of the right nor left insula. This may be a consequence of the duration of our stimulation, which did not exceed $5 \mathrm{~s}$ and lasted $3 \mathrm{~s}$ on average. Still, minor changes in heart rate may have escaped our attention. Following previous reports of connections between the nucleus of the solitary tract and the insula, an involvement of the insula in cortical representation of cardiovascular function is suggested and has been reported in human and animal studies (Hoffman and Rasmussen 1953; Zhang et al. 1998; Abboud et al. 2006). It is possible that these functions may have their cortical representation within the anterior insula, which was less well covered by electrode contacts in this study.

Another difference to previous stimulation studies was that we could not elicit motor phenomena. All motor responses we saw after stimulation of the insula were thought to occur secondarily to sensory phenomena, such as a disgusted facial expression in response to an unpleasant taste phenomenon or touching parts of the body after sensory phenomena in that region. In addition, patients did not report involuntary movements during stimulation of insular electrodes.

None of the symptoms produced by electrocortical stimulation in our study was classified as being a vestibular phenomenon, despite the fact that prominent thalamoinsular afferents arise from the ventral posterior inferior and superior nucleus of the thalamus and progress to the parietoinsular vestibular cortex in the posterior-dorsal insula adjacent to the pain-receptive area (Kahane et al. 2003). Finally, our data are in good agreement with a similar study of invasive electrocortical stimulation in 
patients with epilepsy (Ostrowsky et al. 2000). In this study, symptoms of viscerosensation, somatosensation, nociception and gustation were reported after stimulation of insular electrodes which matched our categories of symptoms. Importantly, viscerosensory and gustatory responses were clearly more often located anterior to painful and non-painful somatosensory symptoms that were found primarily in the posterior insula. Our study reproduces this pattern. Moreover, due to an increased number of electrode contacts implanted by a different technique, we could refine the aforementioned functional categories to more circumscribed insular areas. In contrast to this previous report, we did not find evidence that these functions do extent to the anterior insula; on the contrary, responses were limited to posterior and central insular regions (Ostrowsky et al. 2000). This highlights the possibility that only the granular and dysgranular cortical areas within the human insula encompass cerebral function capable of being excited by electrocortical stimulation. Therefore, our data are in accordance with the heterogeneous cytoarchitecture of the insula.

\section{Limitations}

There are limitations to our study. First, the location of electrodes was determined by superimposition of postoperative CT on preoperative MRI. Hence, there may be a systematic error due to intraoperative shift of the brain prior to obtaining the localizing CT. Nevertheless, in one patient who already had been implanted with insular electrodes, we decided on implantation of additional depth electrodes for diagnostic reasons. We found the position of insular electrodes after both operations to be highly consistent. Second, due to gyration of the insular cortex, a minority of the electrode contacts that were inserted tangential to the insular cortex were in the transitional zone between the grey matter of the insula and the extreme capsule beneath it. Theoretically, responses of such electrodes therefore may not represent insular function but the result of stimulation of fibers of the extreme capsule or fibers of passage. Still the degree of reproducibility of symptoms in our study and the functional continuity to adjacent electrode contacts in the grey matter supports the assumption that the structures stimulated represent functional units. Third, the stimulation intensities used in our study surpass those of similar previous studies using depth electrodes in the insula (Ostrowsky et al. 2000; Isnard et al. 2004). Hence, we cannot exclude that some of the described responses were due to activation of distant cortical areas. Nonetheless, each response reported in our study represents the first symptom that occurred at a single electrode contact, with minimum stimulation intensity, therefore, being a threshold response. In addition, there was no significant difference in stimulation intensities between patients or categories of response, indicating that we did indeed stimulate a common brain structure. The absence of after-discharges at the implanted electrodes and the agreement with previous literature on insular function are further arguments for a primary insular origin of the reported responses. Fourth, since all stimulation was carried out in epileptic patients, results may not be transferable to healthy individuals. The same limitation, however, applies to any previous study of cortical stimulation. Moreover, except of one patient, there was no interictal epileptic activity recorded in any of the insula electrodes. Fifth, the number of patients studied is still very small and individual functional anatomy may have influenced the results disproportionately. On the other hand, the comparably high number of electrode contacts in each insula still allowed a good spatial correlation of evoked symptoms in this study.

\section{Conclusion}

We distinguish four qualitatively and topographically distinct functional areas in the insular cortex. Somatosensory representation in the most posterior part of the insula, a subgroup of thermo- and nociception in the posterior superior insula, viscerosensory responses anterior to the somatosensory area and a subgroup of gustatory responses in the central part of the insula. No responses were detected after stimulation of the anterior insula. These data confirm results from studies in non-human-primates and rodents and refine the functional neuroanatomy of the insula in humans.

Acknowledgments This work was supported by the "Stiftungsrat für die deutsche Wissenschaft" with an educational grant (to C.S.).

Conflict of interest The authors declare that they have no conflicts of interest.

Open Access This article is distributed under the terms of the Creative Commons Attribution Noncommercial License which permits any noncommercial use, distribution, and reproduction in any medium, provided the original author(s) and source are credited.

\section{References}

Abboud H, Berroir S, Labreuche J, Orjuela K, Amarenco P (2006) Insular involvement in brain infarction increases risk for cardiac arrhythmia and death. Ann Neurol 59:691-699

Allen GV, Saper CB, Hurley KM, Cechetto DF (1991) Organization of visceral and limbic connections in the insular cortex of the rat. J Comp Neurol 311:1-16

Bailey P, von Bonin G (1951) The isocortex of man. University of Illinois Press, Urbana 
Benjamin RM, Burton H (1968) Projection of taste nerve afferents to anterior opercular-insular cortex in squirrel monkey (Saimiri Sciureus). Brain Res 7:221-231

Biemond A (1956) The conduction of pain above the level of the thalamus opticus. Arch Neurol Psychiatry 75:231-244

Birklein F, Rolke R, Müller-Forell W (2005) Isolated insular infarction eliminates contralateral cold, cold pain, and pinprick perception. Neurology 65(9):1381

Brodmann K (1909) Vergleichende Lokalisationslehre der Großhirnrinde des Menschen. Barth JA, Leipzig

Brooks JC, Zambreanu L, Godinez A, Craig AD, Tracey I (2005) Somatotopic organisation of the human insula to painful heat studied with high resolution functional imaging. Neuroimage 27:201-209

Cechetto DF, Saper CB (1987) Evidence for a viscerotopic sensory representation in the cortex and thalamus in the rat. J Comp Neurol 262:27-45

Coq JO, Qi H, Collins CE, Kaas JH (2004) Anatomical and functional organization of somatosensory areas of the lateral fissure of the New World titi monkey (Callicebus moloch). J Comp Neurol 476:363-387

Craig AD (2002) How do you feel? Interoception: the sense of the physiological condition of the body. Nat Rev Neurosci 3(8):655-666

Craig AD (2009) How do you feel-now? The anterior insula and human awareness. Nat Rev Neurosci 10:59-70

Craig AD, Bushnell MC, Zhang ET, Blomqvist A (1994) A thalamic nucleus specific for pain and temperature sensation. Nature 372:770-773

Craig AD, Chen K, Bandy D, Reiman EM (2000) Thermosensory activation of insular cortex. Nat Neurosci 3(2):184-190

Dupont S, Bouilleret V, Hasboun D, Semah F, Baulac M (2003) Functional anatomy of the insula: new insights from imaging. Surg Radiol Anat 25:113-119

Eickhoff SB, Schleicher A, Zilles K, Amunts K (2006) The human parietal operculum. I. Cytoarchitectonic mapping of subdivisions. Cereb Cortex 16:254-267

Eickhoff SB, Grefkes C, Zilles K, Fink GR (2007) The somatotopic organization of cytoarchitectonic areas on the human parietal operculum. Cereb Cortex 17:1800-1811

Friedman DP, Murray EA, O’Neill JB, Mishkin M (1986) Cortical connections of the somatosensory fields of the lateral sulcus of macaques: evidence for a corticolimbic pathway for touch. J Comp Neurol 252:323-347

Frot M, Mauguière F (2003) Dual representation of pain in the operculo-insular cortex in humans. Brain 126:438-450

Frot M, Magnin M, Mauguière F, Garcia-Larrea L (2007) Human SII and posterior insula differently encode thermal laser stimuli. Cereb Cortex 17:610-620

Greenspan JD, Lee RR, Lenz FA (1999) Pain sensitivity alterations as a function of lesion location in the parasylvian cortex. Pain $81: 273-282$

Hausser-Hauw C, Bancaud J (1987) Gustatory hallucinations in epileptic seizures. Electrophysiological, clinical and anatomical correlates. Brain 110:339-359

Henderson LA, Gandevia SC, Macefield VG (2007) Somatotopic organization of the processing of muscle and cutaneous pain in the left and rigt insula cortex: a single-trial fMRI study. Pain 128:20-30

Henderson LA, Rubin TK, Macefield VG (2010) Within-limb somatotopic representation of acute muscle pain in the human contralateral dorsal posterior insula. Human Brain Mapping (in press)

Hoffman BL, Rasmussen T (1953) Stimulation studies of insular cortex of Macaca mulatta. J Neurophysiol 16:343-351
Isnard J, Guénot M, Sindou M, Mauguière F (2004) Clinical manifestations of insular lobe seizures: a stereo-electroencephalographic study. Epilepsia 45:1079-1090

Kaas JH (2005) The future of mapping sensory cortex in primates: three of many remaining issues. Phil Trans R Soc Lond B Biol Sci 360(1456):653-664

Kahane P, Hoffmann D, Minotti L, Berthoz A (2003) Reappraisal of the human vestibular cortex by cortical electrical stimulation study. Ann Neurol 54:615-624

Kurth F, Eickhoff SB, Schleicher A, Hoemke L, Zilles K, Amunts K (2010a) Cytoarchitecture and probabilistic maps of the human posterior insular cortex. Cereb Cortex 20:1448-1461

Kurth F, Zilles K, Fox PT, Laird AR, Eickhoff SB (2010b) A link between the systems: functional differentiation and integration within the humans insular revealed by meta-analysis. Brain Struct Funct 214:519-534

Ladabaum U, Roberts TP, McGonigle DJ (2007) Gastric fundic distension activates fronto-limbic structures but not primary somatosensory cortex: a functional magnetic resonance imaging study. Neuroimage 34:724-732

Lenz FA, Rios M, Chau D, Krauss GL, Zirh TA, Lesser RP (1998) Painful stimuli evoke potentials recorded from the parasylvian cortex in humans. J Neurophysiol 80:2077-2088

Mazzola L, Isnard J, Peyron R, Guénot M, Mauguière (2009) Somatotopic organization of pain responses to direct electrical stimulation of the human insular cortex. Pain 146:99-104

Mesulam MM, Mufson EJ (1982a) Insula of the old world monkey. I Architectonics in the insulo-orbito-temporal component of the paralimbic brain. J Comp Neurol 212(1):1-22

Mesulam MM, Mufson EJ (1982b) Insula of the old world monkey. III: Efferent cortical output and comments on function. J Comp Neurol 212(1):38-52

Mesulam MM, Mufson EJ (1985) The insula of Reil in man and monkey. Architectonics, connectivity and function. In: Peters A, Jones EG (eds) Cerebral cortex, vol 4. Plenum Press, New York, pp 179-226

Mufson EJ, Mesulam MM (1982) Insula of the old world monkey. II: Afferents cortical input and comments on the claustrum. J Comp Neurol 212:23-37

Naqvi NH, Rudrauf D, Damasio H, Bechara A (2007) Damage to the insula disrupts addiction to cigarette smoking. Science 315:531-534

Nguyen DK, Nguyen DB, Malak R, Leroux JM, Carmant L, SaintHilaire JM, Giard N, Cossette P, Bouthillier A (2009) Revisiting the role of the insula in refractory partial epilepsy. Epilepsia 50:510-520

Ostrowsky K, Isnard J, Ryvlin P, Guénot M, Fischer C, Mauguière F (2000) Functional mapping of the insular cortex: clinical implication in temporal lobe epilepsy. Epilepsia 41:681-686

Ostrowsky K, Magnin M, Ryvlin P, Isnard J, Guenot M, Mauguière F (2002) Representation of pain and somatic sensation in the human insula: a study of responses to direct electrical cortical stimulation. Cereb Cortex 12:376-385

Penfield W, Faulk ME (1955) The insula; further observations on its function. Brain 78:445-470

Penfield W, Rasmussen T (1950) The cerebral cortex of man. A clinical study of localization of function. MacMillan, New York

Phillips ML, Young AW, Senior C, Brammer M, Andrew C, Calder AJ, Bullmore ET, Perrett DI, Rowland D, Williams SCR, Gray JA, David AS (1997) A specific substrate for perceiving facial expressions of disgust. Nature 389:495-498

Pollatos O, Schandry R, Auer DP, Kaufmann C (2007) Brain structures mediating cardiovascular arousal and interoceptive awareness. Brain Res 1141:178-187 
Pritchard TC, Hamilton RB, Morse JR, Norgren R (1986) Projections of thalamic gustatory and lingual face areas in the monkey. J Comp Neurol 244:213-228

Pritchard TC, Macaluso DA, Eslinger PJ (1999) Taste perception in patients with insular cortex lesions. Behav Neurosci 113:663-671

Reil JC (1809) Die sylvische Grube. Arch Physiol 9:195-208

Riecker A, Gastl R, Kühnlein P, Kassubek J, Prosiegel M (2009) Dysphagia due to unilateral infarction in the vascular territory of the anterior insula. Dysphagia 24:114-118

Rose M (1928) Die Inselrinde des Menschen und der Tiere. J Psychol Neurol 37:467-624

Ruben J, Schwiemann J, Deuchert M, Meyer R, Krause T, Curio G, Villringer K, Kurth R, Villringer A (2001) Somatotopic organization of human secondary somatosensory cortex. Cereb Cortex 11(5):463-473

Ryvlin P, Minotti L, Demarquay G, Hirsch E, Arzimanoglou A, Hoffman D, Guénot M, Picard F, Rheims S, Kahane P (2006) Nocturnal hypermotor seizures, suggesting frontal lobe epilepsy, can originate in the insula. Epilepsia 47:755-765

Saper CB (1982) Convergence of autonomic and limbic connections in the insular cortex of the rat. J Comp Neurol 210:163-173

Singer T, Seymour B, O'Doherty J, Kaube H, Dolan RJ, Frith CD (2004) Empathy for pain involves the affective but not sensory components of pain. Science 303:1157-1162

Small DM (2010) Taste representation in the human insula. Brain Struct Funct 214:551-561

Small DM, Gregory MD, Mak YE, Gitelman D, Mesulam MM, Parrish T (2003) Dissociation of neural representation of intensity and affective valuation in human gustation. Neuron 38:701-711

Starr CJ, Sawaki L, Wittenberg GF, Burdette JH, Oshiro Y, Quevedo AS, Coghill RC (2009) Roles of the insular cortex in the modulation of pain: insights from brain lesions. J Neurosci 29:2684-2694
Stickler D, Gilmore R, Rosenbeck JC, Donovan NJ (2003) Dysphagia with bilateral lesions of the insular cortex. Dysphagia 18:179-181

Türe U, Yaşargil DCH, Al-Mefty RNO, Yaşargil MG (1999) Topographic anatomy of the insular region. J Neurosurg 90:720-733

Valeriani M, Restuccia D, Barba C, Le Pera D, Tonali P, Mauguière F (2000) Sources of cortical responses to painful $\mathrm{CO}(2)$ laser skin stimulation of the hand and foot in the human brain. Clin Neurophysiol 116:1103-1112

Van Buren JM (1983) Sensory responses from stimulation of the inferior Rolandic and Sylvian regions in man. J Neurosurg 59:119-130

Van Buren JM, Borke RC (1972) Variations and Connections of the human thalamus-1: the nuclei and cerebral connections of the thalamus. Springer, Berlin

Vandenbergh J, Dupont P, Fischler B, Bormans G, Persoons P, Janssens J, Tack J (2005) Regional brain activation during proximal stomach distention in humans: A positron emission tomography study. Gastroenterology 128:564-573

Vogt C, Vogt O (1919) Allgemeine Ergebnisse unserer Hirnforschung. J Psychol Neurol 25:279-461

von Economo C, Koskinas GN (1925) Die Cytoarchitectonik der Hirnrinde des erwachsenen Menschen. Springer, Berlin

von Lehe M, Wellmer J, Urbach H, Schramm J, Elger CE, Clusmann $\mathrm{H}$ (2009) Insular lesionectomy for refractory epilepsy: management and outcome. Brain 132:1048-1056

Wang GJ, Tomasi D, Backus W, Wang R, Telang F, Geliebter A, Korner J, Bauman A, Fowler JS, Thanos PK, Volkow ND (2008) Gastric distension activates satiety circuitry in the human brain. Neuroimage 39:1824-1831

Zhang ZH, Dougherty PM, Oppenheimer SM (1998) Characterization of baroreceptor-related neurons in the monkey insular cortex. Brain Res 796:303-306

Zilles K, Amunts K (2010) Centenary of Brodmann's mapconception and fate. Nat Rev Neurosci 11:139-145 